\title{
Hyperbolic Problems: Theory and Computation
}

\author{
Jan S. Hesthaven ${ }^{1}$ • Jae-Hun Jung ${ }^{2}$ • Allen Tesdall ${ }^{3}$
}

Published online: 1 July 2015

C) Springer Science+Business Media New York 2015

This special issue of 'Hyperbolic Problems: Theory and Computation' presents selected papers from the presentations given in the mini-symposium entitled 'Recent Progress in Hyperbolic Problems: Theory and Computation' at the International Conference on Applied Mathematics, Modeling and Computational Science (AMMCS 2013), taking place in Waterloo, Ontario, Canada from August 26 to 30, 2013.

Hyperbolic equations represent a fundamental class of PDEs, used to describe a wide range of natural phenomena such as waves, shocks, flows, and signals, appearing across quantum levels to cosmological scales. During the last several decades, important advances have been made in the theory and in the numerical computation of accurate and stable solutions to these problems.

The mini-symposium aimed to bring together researchers at all levels, providing an opportunity to share and discuss recent progress in both theoretical and computational aspects of hyperbolic problems. In total, 32 presentations were given in the mini-symposium. These presentations covered a broad spectrum of subjects related to problems dealing with hyperbolic PDEs found in various application areas ranging from geophysical problems to large-scale cosmological phenomena. The analysis of theoretical aspects of these problems, together with a diverse set of implementations of numerical methods for their solution, were discussed in the talks.

Jae-Hun Jung

jaehun@buffalo.edu

Jan S. Hesthaven

jan.hesthaven@epfl.ch

Allen Tesdall

allen.tesdall@csi.cuny.edu

1 Ecole Polytechnique Fédérale de Lausanne (EPFL), 1015 Lausanne, Switzerland

2 Department of Mathematics, University at Buffalo, The State University of New York, Buffalo, NY 14260-2900, USA

3 Department of Mathematics, College of Staten Island, City University of New York, Staten Island, NY 10314, USA 
Theoretical questions considered in the presentations included the effect of non-strict hyperbolicity on singularity formation for a quasilinear system, regularity of solutions in a sign-changing Liouville equation, existence of singular shocks in conservation laws with non-classical Riemann solutions, normal form analysis of a Burgers-Hilbert equation, 2D shock reflections, periodic solutions of the Euler equations, well-posedness of 2D wave equations in Sobolev spaces, and dispersive solutions with discontinuous initial data. The computational aspects considered in the presentations covered various numerical methods and their analysis, including recent progress in spectral methods, linear stability, high-order limiters, shock detection and filters for discontinuous Galerkin methods, fast sweeping methods, adaptive CENO and WENO methods, high-order Lagrangian schemes for multi-material compressible flow, and absorbing boundary conditions for quantum wave phenomena. The mini-symposium also featured talks in subjects as varied as parameter estimation and uncertainty propagation in volcanic data, and isentropic flow with large data.

Recently, numerical relativity has become an active and important research area among researchers working on high-order numerical methods for conservation laws. In this research domain, the Einstein field equations are written as a coupled system of elliptic and hyperbolic PDEs in which the elliptic part appears as a constraint equation on the spatial hypersurface, and the hyperbolic part governs the evolution of the fields through the temporal coordinate. The mini-symposium contained five presentations in numerical relativity, including talks on direct numerical simulation of black-hole binaries, spectral methods for helically symmetric gravitational binaries, gravitational and electromagnetic phenomena in strongly gravitating systems, and scalar field evolution in anti-de Sitter universe.

It is impossible to adequately cover the extent of recent advances in theory and computation of hyperbolic problems in a single mini-symposium, or in a special issue. This special issue presents 8 papers, selected with the intent to present frontier and timely research topics and noteworthy new developments in applications for future research.

In this special issue, Chen, Chou and Kao (Lax-Friedrichs Multigrid Fast Sweeping Methods for Steady State Problems for Hyperbolic Conservation Laws) propose to use WENO interpolation at each level of multigrid together with Lax-Friedrichs fast sweeping methods. In this way, the authors obtain highly accurate steady-state solutions efficiently, including for solutions with singularities. The main advantage is that the developed method is more efficient than the regular Lax-Friedrichs fast sweeping WENO method, as it saves the original sweeps. Antoine, Lorin and Bandrauk (Domain Decomposition Method and HighOrder Absorbing Boundary Conditions for the Numerical Simulation of the Time Dependent Schrödinger Equation with Ionization and Recombination by Intense Electric Field) propose a high-order method for absorbing boundary conditions based on the domain decomposition method for solving the time-dependent Schrödinger equation. The system is a multi-scale system where both the nuclei and the free electrons are considered and these different regions are split. It is well known that there exists an inconsistency of accuracy across the split domain interfaces. In this paper, the authors develop an accurate and efficient absorbing boundary condition. The key idea is to design the transmission boundary condition and use a Schwarz waveform relaxation algorithm to reconstruct the transmitted solution in the outside domain where the wave propagates. It is expected that this development will be useful in the numerical simulation of laser phenomena.

In Fast Evaluation of Far-Field Signals for Time-Domain Wave Propagation, Field and Lau construct a mechanism for using solution data for the wave equation on a sphere to compute the solution on a sphere of larger radius, or at infinity. As shown by Greengard, Hagstrom and Jiang, this operation can be expressed in terms of convolution with a sum of exponentials. The exact formula, however, is ill-conditioned for spherical harmonics of large polar index $\ell$. Field 
and Lau use a least squares approximation algorithm (proposed earlier to treat the related problem of applying exact radiation boundary conditions) to replace the exact kernel by an approximating sum that requires fewer exponentials. The authors show numerically that the compressed expressions are much better conditioned than the exact expressions, resulting in a well-conditioned algorithm enabling them to achieve accurate results. In (Hybrid FourierContinuation Method and Weighted Essentially Non-oscillatory Finite Difference Scheme for Hyperbolic Conservation Laws in a Single-Domain Framework) by Li, Gao, Don and Xie, an enhanced hybrid method based on the Fourier-Continuation (FC) and WENO-Z is proposed for solving hyperbolic conservation laws. The new feature in this paper is to combine these two methods in a single domain framework whereas previous FC-WENO hybrid methods utilize a multi-domain framework. The single-domain approach divides the WENO region from the $\mathrm{FC}$ region whenever edges are detected. The paper addresses critical issues related to the implementation of the FC algorithm in hybrid FC-WENO finite difference schemes with uniformly spaced mesh. The numerical experiments show that the resulting scheme is fast, exhibits high resolution, and is essentially non-dissipative and non-dispersive for a long time simulation of shocked flow with fine scale structures. This new algorithm is expected to be an alternative to the multi-domain approach.

Li and Saxton (Non-strictly Hyperbolic Systems, Singularity and Bifurcation) study a $2 \times 2$ non-strictly hyperbolic, parabolically degenerate system introduced by Keyfitz and Kranzer (1983). The authors show that singularities can form at points where strict hyperbolicity is lost. This loss of strict hyperbolicity causes serious analytical difficulties, and the authors develop a Godunov-type numerical method based on exact solutions to Riemann problems obtained by Keyfitz and Kranzer, and present a number of illustrative examples for both smooth and discontinuous data. Tesdall, Sanders and Popivanov (Further Results on Guderley Mach Reflection and the Triple Point Paradox) study a 2D shock reflection problem for the compressible Euler equations. The authors focus on the following open question: does the supersonic patch that forms behind the triple point in the triple point paradox regime have a smooth boundary (so-called Guderley reflection or GR, found in some experiments and computations) or one delimited by a sequence of weak shocks (Guderley Mach reflection or GMR, found in other experiments and computations). They use a third-order method based on quadratic reconstruction from cell averages and approximate Riemann solvers. The solutions obtained provide evidence that GR is simply under-resolved GMR, and is not a distinct reflection geometry.

On the theory side, the article by Jia and Pan deals with an issue to which very little attention has been paid, but which is important from both theoretical and numerical viewpointsthe mathematical justification of the isentropic approximation to the Euler equations. In On Isentropic Approximations for Compressible Euler Equations, Jia and Pan justify the isentropic limit within the regime of classical solutions. They generalize classical results on the Cauchy problem for positive symmetric quasilinear systems to more general Besov space, and establish local well-posedness in Besov space with critical regularity index. Based on this, they obtain an error estimate on the isentropic approximation. The initial entropy is assumed to be a small perturbation about a constant, and the error is proved to be bounded by the perturbation. Temple and Young (A Nash-Moser Framework for Finding Periodic Solutions of the Compressible Euler Equations) provide a clear and concise outline of the authors' program for rigorously establishing existence of space-time periodic shock-free solutions of the compressible Euler equations. This is one of the most intriguing current lines of research within hyperbolic conservation laws. In addition to outlining their approach, the authors provide an illuminating exposition of the power and use of the Nash-Moser technique. 
The guest editors hope that this special issue will provide readers with an interesting glimpse into recent progress in the theory and computation of solutions to hyperbolic problems. They would like to express their gratitude to all the participants in the mini-symposium, and especially to the authors who submitted their valuable papers to this special issue. The reviewers enabled a rigorous review process and provided constructive comments to improve the papers and their time is greatly appreciated. The role of Professor Lilia Krivodonova of the University of Waterloo in the organization of the mini-symposium was crucial, and her help is gratefully acknowledged. Finally, the guest editors thank Editor-in-Chief Chi-Wang Shu of Journal of Scientific Computing, who supported the publication of this special issue and helped to ensure that the issue be completed successfully. 